\title{
O RETRATO DA QUALIDADE DE VIDA DOS GESTORES DA SOCIOEDUCAÇÃO DO DISTRITO FEDERAL: UMA ANÁLISE ESTATÍSTICA DESCRITIVA EM SAÚDE MENTAL
}

\author{
THE PICTURE OF THE QUALITY OF LIFE OF THE SOCIO- \\ EDUCATION MANAGERS OF THE DISTRITO FEDERAL FROM A \\ DESCRIPTIVE STATISTICAL ANALYSIS IN MENTAL HEALTH
}

\author{
Jorge Ponciano Ribeiro ${ }^{1}$ \\ Tatiana de Paula Soares ${ }^{2}$
}

\section{RESUMO}

Este artigo se propôs a identificar e descrever os componentes de saúde física e mental sob os processos de saúde e suas relações ao burnout para avaliar a qualidade de vida da equipe gestora socioeducativa do Distrito Federal. A relevância deste estudo justifica-se pela escassez de pesquisas na área de saúde para este público-alvo e visa retratar os processos, intencionando a promoção de saúde. Como método, utilizou-se o SF-36 Health Survey e o MBI- HSS. Nos resultados, enquanto os índices de saúde física foram elevados, a baixa qualidade de vida foi evidenciada sob o aspecto de saúde mental. Sobre a qualidade de vida relacionada ao desenvolvimento de burnout, quanto maior o esgotamento emocional, mais as relações com a equipe de trabalho foram afetadas. Com relação à saúde mental, quanto maior a 'vitalidade', melhor a saúde mental e quanto menor o esgotamento emocional, maior a qualidade de saúde mental do gestor.

Palavras-chaves: Qualidade de vida; Saúde. Gestores; Sistema socioeducativo.

\begin{abstract}
This article aimed to identify and describe the physical and mental health components of health processes and their relationships to burnout to assess the socio-educational management team's quality of life in Distrito Federal. The relevance of this study lies in the lack of health research with this target audience and aims to portray the processes to promote health. The research method included conducting an SF-36 Health Survey and the MBI-HSS. While we observed high level of physical health indicators, low level of mental health indicators evidenced low quality of life. While physical health indicators were high, there was evidence of the low quality of life due to mental health issues. Regarding the quality of life indicators on burnout development, we found that emotional exhaustion is directly
\end{abstract}

\footnotetext{
${ }^{1}$ Professor Titular Emérito da Universidade de Brasília/DF e da Universidade Estadual de Montes Claros/MG. Doutor em Psicologia pela Pontifícia Universidade Salesiana de Roma, Itália. e-mail: jorgeponcianoribeiro@yahoo.com.br

2 Atualmente é Doutoranda em Psicologia pela Universidade Federal do Rio de Janeiro. Mestra em Ciências Humanas e Sociais com ênfase em Psicologia Clínica, Psicopatologia e Psicologia da Saúde pela Universidade Federal de Toulouse Midi-Pirénées - França, com habilitação em Pesquisa Aplicada e Prevenção em Psicologia da Saúde e Psicopatologia. Mestra em Psicologia Clínica e Cultura pela Universidade de Brasília. e-mail: tatiana.soares@gmail.com
} 


\section{Revista \\ Debates Insubmissos}

linked with the team's work relationships. Concerning mental health, the greater the 'vitality,' the better the mental health, and with less emotional exhaustion, the greater the manager's mental health quality.

Keywords: Quality of life; Health. Managers; Socio-educational system.

\section{INTRODUÇÃO}

O tema do presente estudo são as relações entre qualidade de vida - sob o ponto de vista da saúde -, burnout e a gestão do sistema socioeducativo do Distrito Federal.

A finalidade deste $\operatorname{artigo~}^{3}$ é retratar a qualidade de vida dos gestores do sistema socioeducativo do Distrito Federal sob os processos de saúde e suas relações ao burnout.

De acordo com o Decreto $\mathrm{n}^{\mathrm{o}}$ 37.896, de 27 de dezembro de 2016, o Subsistema do Distrito Federal é gerenciado pela Subsecretaria do Sistema Socioeducativo - SUBSIS, vinculada à Secretaria de Estado de Justiça e Cidadania do Distrito Federal - SEJUS-DF. À SUBSIS compete, de forma geral, planejar, coordenar, executar e avaliar programas, projetos e atividades de medidas socioeducativas e é de sua responsabilidade a gestão e a execução das medidas socioeducativas de Prestação de Serviços à Comunidade (PSC), Liberdade Assistida (LA), Semiliberdade e Internação (DISTRITO FEDERAL, 2016).

Em conformidade à Constituição Federal (BRASIL, 1988), no Estatuto da Criança e do Adolescente - ECA (1990) e no Código Penal (1940), os menores de 18 anos são considerados penalmente inimputáveis. Assim, aos adolescentes que se atribuam autoria de ato infracional, devem ser aplicadas as medidas socioeducativas previstas no Art. 112 do ECA. Tais medidas podem ser aplicadas em jovens de até 21 anos, em caráter excepcional.

Consoante às pesquisas de Baptista (2013), o sucesso de uma política pública tal como a do atendimento socioeducativo depende das delicadas conexões entre diversos atores institucionais devido a sua interdependência. Pequenas falhas nas relações interinstitucionais

\footnotetext{
${ }^{3}$ Este artigo originou-se da pesquisa que compôs a dissertação apresentada ao Instituto de Psicologia como requisito parcial para obtenção do título de Mestre pelo Programa de Pós-Graduação em Psicologia Clínica e Cultura da Universidade de Brasília.
} 


\section{Revista \\ Debates Insubmissos}

podem levar ao seu fracasso. Isso torna o estudo do sistema socioeducativo relevante, pois, neste caso, o governo federal prevê na Lei $\mathrm{n}^{\circ}$ 12.594, de 18 de janeiro de 2012, que os estados federados, os municípios e o Distrito Federal se insiram em um Sistema Nacional de Atendimento Socioeducativo - SINASE, com sistemas estaduais, municipais e distrital.

Acerca da situação do contexto de trabalho dos profissionais do sistema distrital, a literatura revela que são escassos os estudos que a avaliam, quiçá dos gestores, e, tampouco, calcular o seu impacto na execução das medidas socioeducativas. Há ainda menos material quando se trata das vivências de processos de saúde e/ou adoecimento como as prováveis dificuldades dos gestores, impactando no atendimento ao jovem autor de atos infracionais (BAPTISTA, 2013).

Os problemas relativos ao atendimento socioeducativo geram sofrimento para os profissionais do sistema (BAPTISTA, 2013), e entender como se apresentam os processos de saúde dos gestores da socioeducação pode ser crucial para aprimorar a qualidade de vida e a satisfação destes colaboradores no contexto de trabalho. Ao gestor, ao dar-se conta da importância de seu autocuidado, maiores chances terão de atentarem-se à necessidade da promoção de saúde dos trabalhadores sob um olhar coletivo.

Tavares (2004) enfatiza que é a partir da identificação da dinâmica interrelacional no trabalho que se constrói uma proposta reluzindo a priorização e a manutenção da saúde sob a perspectiva de qualidade de vida, assinalando que a percepção de fatores protetivos ao trabalhador pode viabilizar ações coletivas para a promoção de saúde diante das condições ambientais e relacionais do trabalho.

O contexto de trabalho interfere significativamente nas vivências de saúde dos gestores, ou seja, dos trabalhadores que operacionalizam as atividades de gestão

Inexistem pesquisas sistemáticas sobre a qualidade de vida e as condições de trabalho dos educadores sociais que atuam no Sistema Socioeducativo ou bancos de dados sistematizados sobre dados epidemiológicos associados ao adoecimento da categoria no trabalho [...]. Os poucos estudos sobre esse contexto de trabalho, portanto, sinalizam vivências de grande angústia, ansiedade, institucionalização e alienação no trabalho (SOUZA, 2012, p. 83). 
A carência de pesquisas na área de saúde aos trabalhadores do sistema socioeducativo do Distrito Federal (SOUZA, 2012) amplia a relevância deste estudo justamente pela escassez de pesquisas nesta dimensão para este público-alvo e visa retratar os processos de saúde, intencionando a sua promoção.

No que diz respeito à realidade do sistema socioeducativo do Distrito Federal, especialmente aos fatores institucionais e de trabalho, de acordo com Marçal (2007), uma consequência da não disseminação e dainternalização sobre 'como fazer' é a redução do trabalho de gestão a atividades de ordem técnico-burocráticas.

Aos fatores relacionados ao trabalho associados à síndrome de burnout, referenciados pela OMS (WORLD HEALTH ORGANIZATION, 1998) e Maslach (2003), observa-se pouca participação sobre as regras institucionais, alto índice de demandas de trabalho, e condições de trabalho ambientais precarizadas.

Assim, a questão inicial que motivou este artigo foi: Como está a qualidade de vida, sob a ótica de saúde, dos gestores do sistema socioeducativo do Distrito Federal? Consequentemente, os gestores estão vivendo um processo saudável dentro da organização no contexto de trabalho? Como está a percepção de si sob os aspectos de saúde?

O objetivo foi identificar e descrever a qualidade de vida, sob o ponto de vista de saúde, dos gestores do sistema socioeducativo do Distrito Federal, baseado no instrumental SF-36 Health Survey, que apresenta e mensura as condições físicas e psicológicas, e o ambiente no contexto detrabalho. De forma específica, objetivou-se relacionar o índice de esgotamento emocional do questionário Maslach Burnout Inventory Human Services Survey (MBI-HSS) aos domínios de saúde do $S F-36$ para descrever a qualidade de vida dos gestores e verificar se houve diferença significativa entre os níveis de saúde dos gestores de acordo com a hierarquização dos cargos, subdividida em dois subgrupos referentes à coordenação e à operacionalização da política pública em socioeducação.

Como hipótese inicial, pressupôs-se que os gestores apresentam maiores prejuízos em termos de saúde mental quando esgotados emocionalmente. Já a segunda hipótese foi de que, 


\section{Revista \\ Debates Insubmissos}

aqueles que apresentaram domínios mais baixos em sua saúde física e mental, possuíam maior propensão a dificuldades na execução das atividades diárias relacionadas ao ambiente de risco sob insalubridade diante as condições de precariedade institucionais.

Essencial destacar que, embora os materiais utilizados para esta pesquisa trouxeram um panorama da saúde subdivido em subescalas (componentes físico e mental, esgotamento emocional, despersonalização, e realização pessoal), o propósito de análise, ao final, foi integrálos. Com isso, tornou-se passível a discussão de uma análise do indivíduo em suas características multifacetada e unas em que, identificando e relacionando as partes, fez-se possível 'alinhavar' o todo.

O presente estudo buscou verificar características com a intencionalidade de que, a partir da descrição do perfil do gestor e de processos de 'saúde-doença', a autopercepção da realidade interna e os aspectos externos (institucionais) pelos quais convergiam o sentido do trabalho pudessem, de algum modo, vir à tona. Isto posto, este pode ser "o porta estandarte" para se repensar espaços de reflexões atreladas à política pública de saúde mental aos gestores da socioeducação do Distrito Federal.

Na constatação da existência de indícios de que o ambiente de trabalho está adoecido, presume-se que seja maior a possibilidade de piora na execução das competências e atividades de medidas socioeducativas, havendo, consequentemente, interferências negativas no planejamento, na coordenação e na avaliação de programas e projetos. Assim, a baixa qualidade no processo de execução tende a acarretar a ineficácia da política pública, pois as dificuldades da gestão podem levar a uma organização do trabalho engessada e ineficiente, fenômeno este já apontado em trabalhos anteriores para as unidades de execução do sistema (AMARAL, 2008; BAPTISTA, 2013).

Pretendeu-se verificar também se ou quais fatores relacionados ao trabalho, como recursos e disponibilidade de condições e materiais para a execução de tarefas, estavam ligados ou não à qualidade de vida vivenciada pelos gestores, bem como se houve casos de burnout ou não como consequência das demandas relacionadas à precarização de condições ambientais de trabalho. 


\section{A REALIDADE DA SOCIOEDUCAÇÃO BRASILEIRA}

A educação engloba um processo sociocultural que se forma a partir de um leque de tradições, normas e valores que se diferencia a partir de diferentes concepções - filosóficas, políticas e ideológicas (BISINOTO, 2017). É por meio da manutenção e reprodução de paradigmas que ocorre a sua transformação, considerando as relações de poder em cada contexto social. Nesse sentido, a socioeducação insere-se em uma vasta área da educação social, fincada nos direitos humanos, comprometendo-se com a emancipação e a autonomia de cada sujeito em sua comunidade.

Em tese, tendo o território brasileiro como base, a que nos referimos quando falamos em 'socioeducação'?

A socioeducação é um conjunto articulado de programas, serviços e ações desenvolvidosa partir da articulação entre práticas educativas, demandas sociais e direitos humanos com o objetivo de mobilizar nos jovens novos posicionamentos sem, contudo, romper com as regras éticas e sociais vigentes (BISINOTO et al., 2015, p. $583)$.

De acordo com Soares, Silva e Antun (2014) e Silva (2017), a prática socioeducativa foca na promoção de estímulos e incentivos à cultura e às possibilidades de acesso a serviços públicos para "[...] que com a identificação por parte dos profissionais, em conjunto e por meio do (re)conhecimento dos jovens, ocorra o 'despertar' de sonhos e de desejos; e, o avistar de habilidades e dons específicos para novas possibilidades de (re)integração social [...]" (SOARES, SILVA; ANTUN, 2014, p. 13). Nessa ótica, Jesus (2013) acrescenta que as medidas socioeducativas possuem, sim, um caráter sancionatório, entretanto, o foco da ação deve pautarse em instrumentos pedagógicos.

Sobre o panorama institucional onde atuam os gestores da socioeducação, alvo deste estudo, destaca-se que a SUBSIS, pautada nas diretrizes do SINASE, deve focar em ações além da responsabilização do jovem, visando o resgate da convivência familiar, o incentivo ao estudo, o fortalecimento de vínculos comunitários e o estímulo à autonomia por meio da participação social.

Desse modo, emergiu a necessidade de investigação científica das ações dos gestores desta subsecretaria e como estão as suas vivências na organização, aqui, em especial, no 


\section{Revista \\ Debates Insubmissos}

tocante à saúde. Faz-se ainda necessário tornar visível a repercussão dessas ações na efetivação da execução da política pública socioeducativa.

No tocante aos aspectos de saúde dos profissionais neste contexto institucional, Ribeiro (2019) elenca fatores fundamentais que norteiam o desenvolvimento de relações socioprofissionais saudáveis, sendo eles: considerar a saúde sob qualquer forma de contato a partir de uma visão sistêmica e integrada, incluir os profissionais e a comunidade atendida como figura central do programa de atendimento, estimular a corresponsabilidade aliada à equanimidade da competência, usar de modo adequado a linguagem dos líderes, neste caso, dos gestores, quer seja diante da assistência interna ou externa ao usuário do serviço e ainda promover a saúde, de forma não reducionista, para amenizar sintomas.

"A grande questão é: bastam um curso, um programa, para introduzir uma nova atitude, ou é o coração e a mente das pessoas que têm de ser atingidos ou até mudados?" (RIBEIRO, 2019, p. 171). Faz-se essencial a atenção aos aspectos individuais, englobando a integralidade do indivíduo ao que permeia sua personalidade, seu comportamento, sentimento, sensação, contexto social e espiritual; paralelo à contínua revisão identitária na construção e nas vivências dos valores, cultura e atitudes da organização.

Com contribuições críticas apontando a realidade da socioeducação brasileira, Souza (2012), em sua tese de doutorado, enfatiza que "[...] a saúde dos profissionais dos programas de atendimento socioeducativo é tema secundário nas pesquisas atuais e não existem dados epidemiológicos sistematizados sobre os processos de adoecimento no trabalho para essa população [...]" (SOUZA, 2012, p. 7), menos ainda dados de saúde voltados para gestores que, em tese, dedicam-se para pautar a socioeducação brasileira fundamentalmente no SINASE (2012) e no I Plano Decenal de Atendimento Socioeducativo - PDASE, elaborado em 2016.

Com base em revisão sistemática e em fontes de pesquisas acadêmicas ${ }^{4}$, verificou-se, em uma perspectiva de gênero, que no sistema socioeducativo brasileiro não constavam

\footnotetext{
${ }^{4}$ Pesquisaram-se os tópicos (assuntos) - "Mulheres na gestão da administração pública", "Mulheres e gestão na administração pública", "Trabalho Feminino", "Mulheres", "Gênero", "Gestão", "Socioeducação", "Women in
} 


\section{Revista \\ Debates Insubmissos}

definições e práticas de gestão desenvolvidas - e desenhadas - por e para mulheres que gerenciavam o atendimento socioeducativo.

Todavia, ressalta-se que esta pesquisa não destacou substancialmente as diferenças e as primazias levantadas por questões de gênero; de forma geral, pretendeu diminuir a escassez dos estudos sobre o contexto de saúde no sistema socioeducativo do Distrito Federal, aproximandose de estudos já realizados pela Universidade de Brasília, mas, de forma pioneira, colocando como objeto principal os gestores da socioeducação do Distrito Federal.

Com isso, urgiu a necessidade de questionar, com intento menos de solucionar e mais de refletir: Qual o perfil dos "líderes" da gestão da socioeducação? Quem são essas pessoas? Como elas se veem? Qual o nível de satisfação e realização no ambiente de trabalho? São satisfatórias as suas relações neste ambiente? Como é abrangida a sua saúde neste contexto? As pessoas que lideram a gestão estão esgotadas emocionalmente? Há um processo de autocuidado e de amparo institucional em relação as suas próprias vivências de saúde?

\section{SAÚDE SOB OS ASPECTOS DE QUALIDADE DE VIDA E O BURNOUT}

Nesta década, em especial nos últimos cinco anos, muito se tem estudado sobre a organização do trabalho, mas pouco dispomos de dados clínicos que amparem o gestor nas dificuldades enfrentadas em seu cotidiano sob o ponto de vista da saúde.

Sob a ótica da psicossomática, Bion (1988) ressalta que o psíquico responde ao corporal e vice-versa, havendo, portanto, um sistema onde não se delineia uma nítida divisão entre ambos. Desse modo, as injunções sociais atuam sobre este aparato complexo que éo ser humano. Saúde implica estilo e ritmo de vida culturais, modalidade da organização do trabalho, vida urbana e tantos outros fatores, remetendo a uma suposta unidade "socio-psicossomática".

Para tecer considerações sobre a definição de saúde dada pela Organização Mundial da Saúde (WORLD HEALTH ORGANIZATION, 2017) a partir do Health Council of the

\footnotetext{
public administration management", "Women in public administration", "Women", "Management", "Gender",
} "Social Education”, “Women’s work”, em 2018. 


\section{Revista \\ Debates Insubmissos}

Netherlands em 2011, há a necessidade de se discutir, o conceito de qualidade de vida. Sob uma compreensão mais dinâmica, os indicadores que englobam a definição de saúde dimensionam as categorias física, mental, espiritual, social, qualidade de vida e atividades da vida diária (LOBÃO, 2019).

Dentro da bioética, segundo Bion (1988), tem-se o conceito de "autonomia", que compreende a qualidade de vida como algo intrínseco, somente passível de ser avaliado pelo próprio sujeito. Prioriza-se a subjetividade uma vez que, de acordo com o conceito de Bion (1988), a realidade é de cada um. Não há rótulos de boa ou má qualidade de vida, embora a saúde pública, para a elaboração de suas políticas, necessite de seus indicadores.

Assim, consideramos imprescindíveis, dentro de uma sociedade, estatísticas que apontem índices de saúde-doença. De modo geral, a doença não pode ser definida somente sob análises estatísticas. Para sua definição, faz-se mister considerar aspectos físicos, mentais, sociais e os que situarem fora da, assim chamada, "normalidade".

O adoecimento é um processo que se desenvolve em um continuum e é concebido ao longo de um eixo temporal do qual se estabelecem os confrontos entre força vital e força desestabilizadora. É um processo de interação continuada, que é o processo saúde-doença. Saúde mental é uma dimensão associada desse processo, necessariamente visto em seu todo. Não existe adoecimento mental que se constitua isoladamente do corpo e das interrelações humanas. Saúde mental não pode ser confundida com adaptação (CANGUILHEM, 1990, p. 257).

A Organização Mundial de Saúde (WHOQOL GROUP, 1994) considera que, para viver uma 'boa saúde', faz-se necessário basicamente o acesso a serviços de prevenção e assistência médica de qualidade, ter um padrão financeiro que proporcione qualidade de vida, educação e vida social saudável, contar com reservas naturais de qualidade, água potável, ar com baixos índices de poluição, trabalho em condições adequadas e segurança, considerar aspectos hereditários - genéticos, atentar-se a uma rotina que trate questões ligadas a bebidas alcoólicas, outras substâncias químicas e dificuldades psicológicas, e prática de atividades físicas (SOUZA et al., 2019).

Uma boa percepção da saúde é importante para avaliar se o quadro de saúde da população é satisfatório, além de poder definir se as intervenções de assistência à saúde estão 
sendo benéficas. Vale destacar a premissa da OMS que afirma que "[...] saúde é um estado de completo bem-estar físico, mental e social e não apenas a ausência de doença ou enfermidade [...]" (WORLD HEALTH ORGANIZATION, 1986).

No Brasil, a saúde é um dos direitos à cidadania presentes na Constituição da República Federativa do Brasil (1988) - apresentado nos artigos 196, 197, 198 e 199. A legislação remete à ideia de uma "saúde ótima", possivelmente inatingível e utópica, já que a mudança e a não a estabilidade são predominantes na vida. Sob este prisma, saúde não é um “estado estável” que, uma vez atingido, possa ser mantido.

Saúde é contato, contato é saúde. Qualquer interrupção disfuncional do contato implica uma perda na saúde. Contato é o processo de autorregulação organísmica, é ajustamento criativo que decorre, sobretudo na diferenciação organismo/ambiente [...]. Doença significa interrupção do contato em um dos quatro campos que compõem o espaço vital da pessoa: geobiológico, psicoemocional, socioambiental e sacrotranscendental. (RIBEIRO, 2019, p. 24).

Sob o tecido gradual da constituição de processos de saúde, Zanello (2018) acrescenta que a 'criação de tipos humanos' interpela as identidades e, de forma simultânea, as práticas institucionais e mudanças materiais estão interligadas a esse processo de construção de identidade.

O discurso médico-científico não contempla, portanto, o significado mais amplo da saúde e do adoecer. Em contrapartida, a definição de qualidade de vida pela OMS (2017) está introduzida na saúde. A qualidade de vida possui um conceito amplo que compreende a saúde física, o estado psicológico, o nível de independência e as relações sociais do indivíduo e suas interfaces com o meio onde está inserido, abrangendo sua percepção quanto à posição que ocupa no contexto da cultura e dos sistemas de valores em que está envolvido, bem como os seus objetivos, expectativas, preocupações e padrões (WHOQOL GROUP, 1994; FLECK, 2000; SEIDL, ZANNON, 2004).

De acordo com Coutinho e Franken (2009), a compreensão sobre o que é qualidade de vida deve nortear ofertas de serviços às políticas públicas como um exercício de cidadania e de atendimento a um direito individual. Faz-se mister a disseminação social para desmitificar o conceito de qualidade de vida associado ao mito de felicidade e buscar como recurso em 


\section{Revista \\ Debates Insubmissos}

organizações a percepção da saúde do trabalhador como diretamente proporcional ao seu nível de produtividade.

Ribeiro (2019) enfatiza ainda que, sob o aspecto da saúde, a organização é natural ao organismo e a desorganização é patológica. Nesse contexto, Souza (2012, p. 7), especificamente ao contexto socioeducativo alude que:

A saúde dos educadores sociais é tema que emerge indiretamente e que remete a relatos sobre a precariedade das condições e dos meios de trabalho, sobre a baixa qualidade de vida no ambiente de trabalho, bem como sobre os principais sintomas percebidos fora da unidade socioeducativa.

E o burnout? Em que condiz com processos de saúde e de qualidade de vida?

Burnout é um termo proveniente da língua inglesa (burn + out) utilizada para sintetizar queima total - na engenharia aeronáutica. Nas ciências sociais é sinônimo de desgaste humano. No jargão popular inglês, refere-se àquilo que deixou de funcionar por absoluta falta de energia. Enfim, é uma metáfora para significar aquilo, ou aquele, que chegou ao seu limite e, por falta de energia, não tem mais condições de desempenho físico ou mental (BENEVIDESPEREIRA, 2012). Ainda para essa autora, o termo burnout foi introduzido para se referir a um fenômeno observado entre os trabalhadores de serviços humanos que precisavam lidar com indivíduos emocionalmente exigentes.

O Guidelines for the Primary Prevention of Mental, Neurological and Psychosocial disorders: Staff Burnout (WORLD HEALTH ORGANIZATION, 1998) abrange os seguintes componentes do burnout: a exaustão emocional que envolve os sentimentos de desesperança, solidão, tristeza, raiva, impaciência, irritabilidade, tensão, sensação de baixa energia, fraqueza e preocupação; a possibilidade de estar associada a cefaleias, náuseas, tensão muscular, dor lombar ou cervical e distúrbios do sono; o distanciamento afetivo, denominado ‘despersonalização', que provoca a sensação de alienação em relação aos outros, sendo a presença destes, muitas vezes, desagradável e não desejada; já o último componente é representado pela baixa realização profissional ou a baixa satisfação com o trabalho, descrito como uma sensação de que muito pouco tem sido alcançado e o que é realizado não tem valor. $\mathrm{O}$ trabalhador não vê mais sentido na sua relação com o trabalho, de forma que já não importa 


\section{Revista \\ Debates Insubmissos}

mais fazer qualquer esforço pois tudo já lhe parece não fazer sentido (CODO; VASQUESMENEZES, 1999).

Os profissionais mais suscetíveis ao desenvolvimento da síndrome de burnout são os de natureza assistencial (BENEVIDES-PEREIRA, 2012) e trabalham diretamente em contato com outras pessoas, muitas vezes cuidando delas, como no caso dos profissionais de saúde, ou tendo simplesmente que lidar com elas, como no caso dos profissionais de educação.

E como enfrentar o esgotamento e o desenvolvimento da Síndrome de burnout?

A ação política, para Alvim (2020), é base para a constituição de 'espaços de saúde'. Esta ação precisa ser feita para o campo (contexto socioeducativo), para o coletivo (gestores) e que atende ao bem comum (comunidade socioeducativa). Somente a partir da constituição de espaços coletivos de fala de modo a 'descristalizar' o processo engendrado de 'silenciamento' da exclusão e desigualdade é que poderá acionar as necessidades do 'corpo' (ALVIM, 2020), vislumbrando, assim, perspectivas outras de 'vir a ser' sob dinâmicas diversas.

Retomando ao objetivo deste estudo, o método colocado a seguir trouxe luz às perguntas: $\mathrm{O}$ esgotamento emocional interferiu na saúde física e/ou mental dos gestores? $\mathrm{O}$ estado de saúde variou conforme a hierarquização de cargos, de acordo com a estrutura organizacional das funções comissionadas? Em que medida esses gestores têm se apresentado sob estado/aspectos de vitalidade e cansaço? Em que proporção a saúde psíquica interferiu na execução do trabalho cotidiano dos gestores? E as condições físicas interferiram na execução das atividades diárias?

\section{METODOLOGIA}

Para a avaliação da qualidade de vida, o SF-36 Health Survey foi o instrumento escolhido por ser o mais utilizado para o uso em prática clínica e pesquisa, evolução em políticas de saúde e pesquisas para a população em geral (BOWLING; BRAZIER, 1995). E para correlacionar a qualidade de vida e o desenvolvimento de burnout, selecionou-se o Maslach Burnout Inventory-Human Services Survey - MBI-HSS (MASLACH, 2003), 


\section{Revista \\ Debates Insubmissos}

questionário também conhecido mundialmente com fins específicos paras investigações sobre burnout. Ambos os instrumentos são validados no Brasil.

Ainda que o processo metodológico aqui utilizado seja de natureza estatística descritiva, este estudo intencionou identificar aspectos de saúde, sob o $S F-36$, não para 'desmembrar' características de saúde evidenciadas pelos gestores no contexto da socioeducação, mas sim para apresentá-las sem perder de vista a interação, a correlação e a interdependência no sentido uno do funcionamento do organismo.

O convite para a participação junto aos gestores se deu pela plataforma online LimeSurvey via eletrônica (e-mail) pela atual Coordenação de Políticas e Atenção à Saúde de Jovens e Adolescentes da SEJUS-DF.

O total de respondentes que concluíram completamente todas as 105 perguntas e questionários foi de 48 do universo de $218^{5}$ gestores, sendo que 103 iniciaram o preenchimento dos itens.

A amostra foi validada considerando a representatividade do número total de entrevistados que preencheram todos os instrumentos de pesquisa. $\mathrm{O}$ estudo, de forma global, abrangeu três instrumentos que avaliam a saúde relacionada ao contexto de trabalho, quer seja para avaliação do desenvolvimento de burnout, de domínios de saúde física e mental quer seja para avaliação de escalas que identificam a satisfação no trabalho. Além disso, a pesquisa contou com perguntas sociodemográficas e com questões relacionadas aos fatores de risco para o burnout.

Neste artigo, o foco da análise ${ }^{6}$ se deu sobre o SF-36 Health Survey (BOWLING; BRAZIER, 1995) para descrever a qualidade de vida dos gestores. O questionário SF-36 Health

\footnotetext{
${ }^{5}$ Dados fornecidos pela atual Coordenação de Políticas e Atenção à Saúde de Jovens e Adolescentes da Subsecretaria do Sistema Socioeducativo da Secretaria de Estado de Justiça e Cidadania do Distrito Federal em 2019.

${ }^{6}$ A Base e a Coleta de Dados desta pesquisa foram as mesmas utilizadas para embasar os seguintes estudos: Master de Sciences Humaines et Sociales, Mention Psychologie Clinique, Psychopatologie et Psychologie de La Sante da Universite Toulouse II, e Mestrado do Instituto de Psicologia do Programa de Pós-Graduação em Psicologia Clínica e Cultura da Universidade de Brasília.
} 


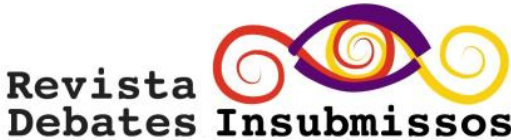

Survey possui 36 (trinta e seis) itens, 8 (oito) subescalas que agregam de 2 a 10 itens cada e 2 (duas) medidas sintetizadas que agrupam as subescalas. As duas medidas do SF-36 Health Survey caracterizam-se como escalas que sintetizam o componente físico $(P C S)$ e o componente mental (MCS). O SF-36 Health Survey avalia a frequência dos domínios: capacidade funcional, limitações por aspectos físicos, dor, quadro geral da saúde, vitalidade, aspectos sociais, limitações por aspectos emocionais e saúde psíquica. Estes fatores variam de 0 a 100 , onde $0=$ pior e 100 = melhor para cada domínio.

Como parte inicial da análise, foi traçado um perfil sociodemográfico, profissiográfico e psicossocial dos participantes visando identificar as principais características dos gestores. A partir daí, foram observados os dados relacionados aos fatores psicológicos, como tratamentos psicoterápicos ou outro tipo de apoio emocional, e destacados os aspectos institucionais referentes ao desenvolvimento de burnout.

$\mathrm{Na}$ avaliação estatística de saúde física $(P C S)$ e mental $(M C S)$ dos participantes estudados, utilizou-se regressão linear múltipla, com o intuito de encontrar relações entre o desenvolvimento de burnout - ligado aos aspectos institucionais, medido pela escala do $M B I-$ com as medidas de saúde citadas (verificadas pelo $S F-36$ ). As variáveis dependentes foram $P C S$ e $M C S$, e as independentes foram MBI-EE (escala de esgotamento emocional), $M B I-D E$ (escala de despersonalização) e $M B I-R P$ (escala de realização pessoal).

Dado que um dos objetivos da pesquisa foi avaliar se houve diferença significativa entre os níveis de saúde de acordo com os cargos ocupados pelos gestores, utilizou-se, então, o teste não paramétrico de Wilcoxon-Mann-Whitney. Além disso, o teste de Kruskal-Wallis foi usado para comparar saúde física ou mental e relações sociais no trabalho.

De antemão, sob autorização da Secretaria de Estado de Justiça e Cidadania do Distrito Federal, o convite e o Termo de Consentimento Livre e Esclarecido para participação desta 
pesquisa foi enviado por esta organização, via correio eletrônico, aos gestores deste sistema. Os dados foram analisados pelo software livre R-3.6.1 (R CORE TEAM, 2019).

Em seguida, as análises e os resultados são evidenciados para elucidar as hipóteses e os objetivos apresentados anteriormente no estudo.

\section{ANÁLISE DOS RESULTADOS}

Para traçar o perfil da gestão do sistema socioeducativo do Distrito Federal a partir de dados sociodemográficos, profissiográficos e questões psicossociais, dois grupos foram criados, de acordo com a hierarquização de cargos.

O Grupo 1, composto por 12 participantes, sendo 8 mulheres e 4 homens, referiu-se aos gestores sob posições hierárquicas de execução para coordenação, planejamento e gestão, podendo abarcar as funções de subsecretário, coordenador, chefe e diretor. Neste Grupo, a idade média foi de 39 anos, sendo que 9 possuíam o estado civil como 'solteiros(as)' e 3 se consideraram em 'algum tipo de relacionamento'.

O Grupo 2, formado por 36 gestores, a saber: 24 mulheres e 12 homens, abrangia 23 'solteiros(as)' e 13 'em um relacionamento'. Este Grupo referiu-se aos cargos hierárquicos de execução operacional de gestão e assessoria, podendo conter as funções de vice-diretor, gerente, assessor, assessor da escola, chefe de plantão e supervisor. Para este Grupo, a idade média foi de 40 anos.

Sobre suas atividades extras às da secretaria (realizadas pelos 48 participantes), $66 \%$ informaram exercer as de cunho doméstico e $37 \%$ àquelas vinculadas à educação dos filhos. Já $31 \%$ dos participantes se ocupavam em outros cursos e $18 \%$ em outros empregos. Cerca de $60 \%$ consideraram-se 'parcialmente satisfeitos' com o tempo empregado em suas atividades de lazer, cultura, esporte e/ou arte. Somente $22 \%$ relataram estar plenamente satisfeitos e $16 \%$ não estavam satisfeitos com o tempo direcionado para diversão, cultura, atividades esportivas e/ou artísticas. 
Esses dados foram importantes para ponderar que os resultados de saúde apontados pelo SF-36 puderam ser influenciados também pelas atividades desgastantes além do desempenho da função no ambiente de trabalho. A maior parte dos gestores não se encontraram satisfeitos com o tempo dedicado às atividades consideradas 'não estressantes'. Além disso, as atividades mais realizadas fora do trabalho no sistema socioeducativo foram caracterizadas por alta sobrecarga, destacando-se o serviço doméstico.

Após a aplicação do questionário $S F-36$, verificou-se se tais medidas foram influenciadas pela hierarquização de cargos.

Figura 1- Comparação do estado de saúde física (PCS) e mental (MCS) entre os dois grupos avaliados

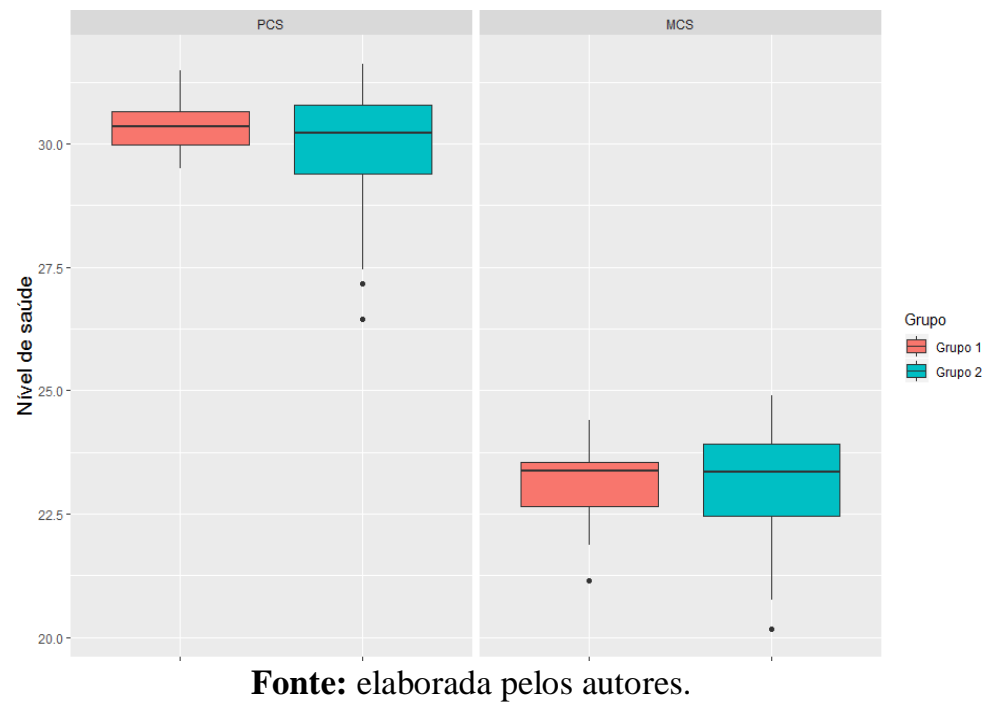

A Figura 1 comparou os níveis de saúde física e mental, calculados a partir do questionário $S F-36$, entre os cargos de maior responsabilização, conforme atribuições dos respectivos cargos referentes ao Grupo 1 e ao Grupo 2. As caixas, em posições próximas nos dois aspectos de saúde, indicaram que não houve diferença significativa entre os dois grupos de gestores, de acordo com a proporcionalidade das respectivas medianas (participação da metade dos participantes de cada grupo, relacionados aos níveis de saúde - PCS e $M C S)$. O Teste de Wilcoxon-Mann-Whitney apresentou a mesma conclusão $(p=0,533$ e $p=0,488$ para $P C S$ e $M C S$, respectivamente). Entretanto, a diferença entre os níveis de saúde física e mental foram 
notoriamente desiguais. Ambos os grupos apresentaram índices maiores de saúde física e mostraram baixa qualidade de vida sob o aspecto da saúde mental.

Destaca-se que, para fins de interpretação, o $S F-36$ possui a finalidade descritiva dos processos de saúde, em termos de qualidade de vida, e não conclusiva de diagnóstico.

Tabela 1 - Medidas descritivas para os oito domínios do SF-36 dos dois grupos de hierarquia

\begin{tabular}{lcccccccc}
\hline & CF & LF & DO & SG & VT & AS & LE & SP \\
\hline Média & 92 & 73 & 69 & 79 & 55 & 62 & 70 & 70 \\
IC $(95 \%)$ & $90 ; 94$ & $69 ; 78$ & $63 ; 75$ & $75 ; 83$ & $52 ; 57$ & $58 ; 66$ & $66 ; 75$ & $68 ; 73$ \\
\hline \multirow{7}{*}{ Média } & 86,4 & 77,6 & 68,5 & 69,4 & 54,6 & 58,0 & 73,8 & 70,6 \\
IC $(95 \%)$ & & 73,$8 ; 81$, & 65,$0 ; 72$, & 66,$1 ; 72$, & 52,$6 ; 56$, & & 69,$9 ; 77$, & 68,$7 ; 72$, \\
& 83,$5 ; 89,3$ & 4 & 0 & 7 & 6 & 54,$9 ; 61,1$ & 8 & 5
\end{tabular}

Nota. CF - Capacidade funcional; LF - limitações por aspectos físicos; DO - dor; SG - quadro geral da saúde VT- vitalidade; AS - aspectos sociais; LE - limitações por aspectos emocionais; SP - saúde psíquica.

Fonte: elaborada pelos autores.

Para o SF-36, os dois estados de saúde (física e mental) são variáveis que dependem dos outros domínios. A Tabela 1 mostrou a comparação entre os dois grupos hierárquicos observando os níveis dos oito domínios desenvolvidos pelo $S F-36$. A capacidade funcional (CF) apresentou um nível médio maior para o Grupo 1, e a estimativa de erro da média pôde variar em até quase 95 mostrando que, em geral, o Grupo 1 possui ótima capacidade funcional, dado que o máximo é 100. Entretanto, o escore médio da vitalidade (VT) apresentou um índice significativamente menor que o domínio da capacidade funcional. Além disso, o domínio (VT) foi equivalente em ambos os Grupos.

Com relação aos domínios específicos de saúde psíquica (SP) e de dor (DO) também não houve diferença relevante entre os dois Grupos.

Dos participantes com menores índices de vitalidade ( $n=14$, com escore abaixo de 45), cerca de $36 \%$ deles afirmaram passar por tratamento psiquiátrico e $93 \%$ destes 14 participantes concordaram que "há riscos no ambiente de trabalho, tais como calor, frio e ruídos excessivos ou iluminação insuficiente, pouca higiene, alto risco tóxico e até de vida". Para os que possuíram um índice maior de vitalidade, esse percentual diminuiu para 85\%, confirmando 


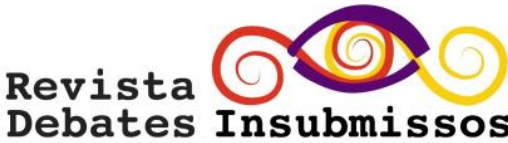

então a segunda hipótese deste artigo quanto à relação entre as quatro variáveis: $P C S, M C S$, VT e ao item "Quanto à normatização (regras), você considera: g) que há riscos em seu ambiente físico, tais como: calor, frio e ruídos excessivos ou iluminação insuficiente, pouca higiene, alto risco tóxico e até de vida". Este item refere-se ao número 23 do questionário profissiográfico.

Aqueles gestores que obtiveram índices baixos em relação à qualidade da saúde, sob o ponto de vista do $S F-36$, apresentaram mais dificuldades na execução das atividades diárias devido aos riscos no ambiente de trabalho. É importante observar que o nível de escore médio da vitalidade (VT) foi o menor dentre os oitos domínios, independentemente da hierarquia entre os cargos.

O quadro geral da saúde (SG) apresentou índice médio de 10 pontos a menos no Grupo 2 com relação ao Grupo 1, e essa diferença foi mantida também no Intervalo de Confiança. Com isso, observou-se que os participantes que executavam funções operacionais de gestão correspondentes ao Grupo 2, ainda que com a taxa de 10 pontos menor em relação ao Grupo referente às posições hierárquicas de execução para coordenação, planejamento e gestão, relacionadas ao Grupo 1, não revelaram diferenças significativas entre eles. Salienta-se ainda que estes dados supostamente 'equivalentes' para ambos os grupos não mensuraram as consequências de saúde, pois o Grupo 2 possui a característica de ser três vezes maior no número de participação - considerando que havia mais disponibilidade de cargos para as funções correspondentes a este Grupo. Ou seja, aqui se evidenciou somente a pontuação de acordo com a hierarquização de cargos.

A maioria dos domínios apresentaram índices satisfatórios nos dois Grupos,com exceção dos domínios sobre vitalidade e aspectos sociais. Contudo, quando calculados todos os índices das subescalas de saúde física e mental e integrando-os de acordo com a fórmula referente à análise do $S F-36$ - referente à qualidade de vida dos participantes -, obtém-se, em uma escala de 0 a 100 pontos, 72.02 como resultado dessa apuração. Entretanto, para identificação precisa correspondente a cada subescala de saúde física e saúde mental, é legítimo considerar as respectivas medidas, de acordo com as pontuações correspondentes a cada uma delas, em que a saúde mental se mostrou visivelmente mais baixa que a saúde física. 


\section{Debates Insubmissos}

Revista

Figura 2 - Comparação entre saúde mental $(M C S)$ e estresse ao trabalhar com pessoas $(M B I)$

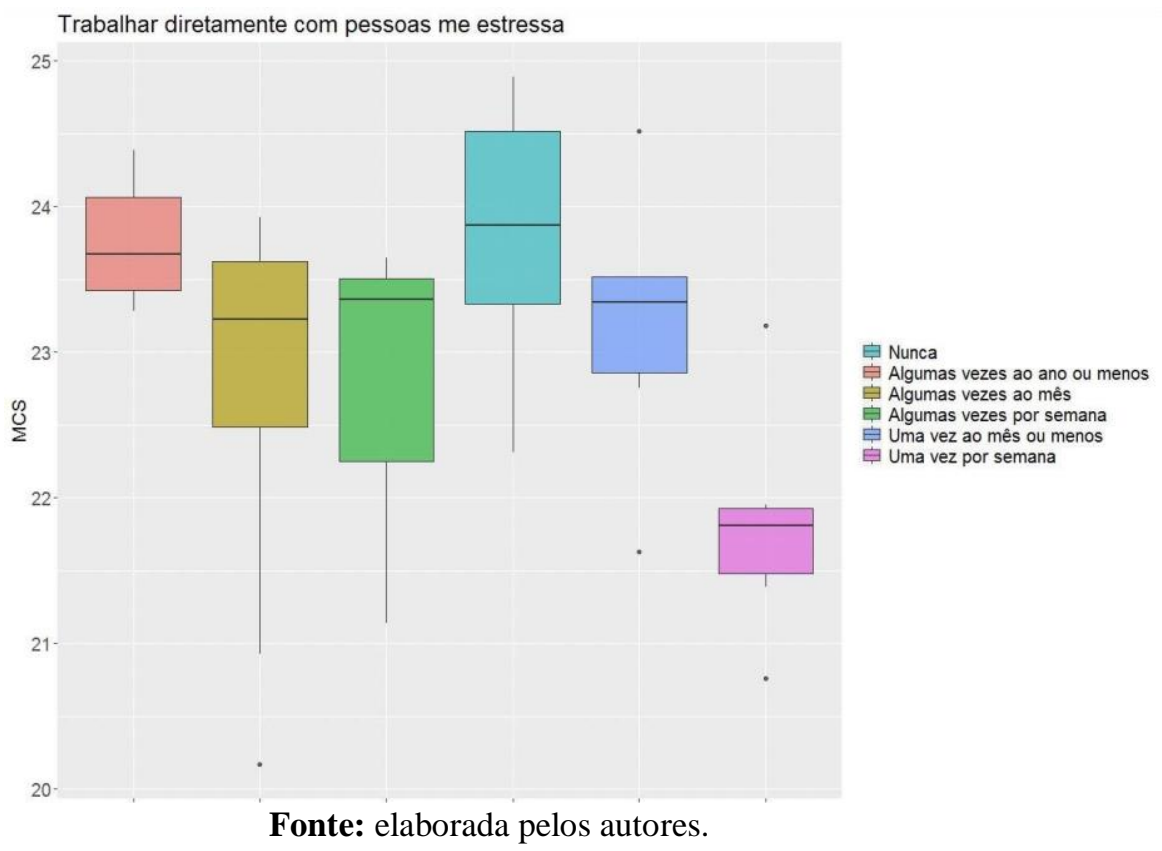

Em conformidade ao item 16, 'Trabalhar diretamente com pessoas coloca muito estresse

em mim', do Maslach Burnout Inventory Human Services Survey (MBI-HSS), correspondente à avaliação do nível de esgotamento emocional (MASLACH, 2003), pela Figura 2 foi notório que os participantes que afirmaram que nunca se estressaram ao lidar diretamente com pessoas mostraram uma saúde mental melhor que todos os demais. Já aqueles que se estressaram 'uma vez por semana' possuíram o pior quadro de saúde mental. De forma complementar, o teste Kruskal-Wallis apresentou uma relação de dependência entre esse tipo de característica de esgotamento emocional e de saúde mental $(p=0,035)$.

Outros indicativos de qualidade de vida foram observados entre 'esgotamento emocional' (MBI-HSS) e 'saúde mental' (SF-36) e entre 'esgotamento emocional' (MBI-HSS) e 'vitalidade' (SF-36). As duas comparações, de acordo com a correlação linear de Pearson, mostraram correlações inversas, ou seja, quanto maior uma medida, menor a outra. Por exemplo, entre 'esgotamento emocional' e 'saúde mental' (MCS), a relação foi igual a -0,53, apontando que quanto maior o 'esgotamento emocional' (MBI-EE), pior a 'saúde mental' (MCS) do participante, assim como quanto menor o 'esgotamento emocional' (MBI-EE), mais vitalidade (SF-36, VT) 


\section{Revista \\ Debates Insubmissos}

têm os participantes, porém, com uma correlação mais forte que a anterior $(=-0,7524)$. Assim, confirmou-se a primeira hipótese deste artigo, ao apontar que 'gestores com nível de esgotamento emocional mais elevado' possuíram a saúde mental mais prejudicada.

\section{DISCUSSÃO E CONSIDERAÇÕES FINAIS}

O status de saúde é um construto social no qual a avaliação de uma pessoa é feita dentro de uma compreensão cultural específica sobre o que se acredita ser saudável. A validade transcultural de um instrumento deve adotar o sistema descritivo desenvolvido internacionalmente. Nessa direção, o propósito macro do presente artigo foi apresentar a qualidade de vida a partir da descrição e das correlações das escalas e das medidas, em especial do $S F-36$.

Com as análises correlacionais que compararam a distribuição em grupos interdependentes, de acordo com Ferreira e Mendes (2003), neste estudo apontou-se o panorama inicial com dados quantitativos que podem contribuir e enriquecer pesquisas futuras sob a fundamentação hermenêutica e qualitativa. Em relação à questão central que buscou identificar a qualidade de vida dos gestores do sistema socioeducativo do Distrito Federal sob o $S F-36$, em síntese, evidenciou-se que a qualidade da saúde dos gestores mostrou-se semelhante nos dois grupos subdividos neste estudo ( $P C S, p=0,533$ e $M C S, p=0,488$ ) e, conforme evidenciou-se nos resultados citados, com níveis de medida diferentes nos aspectos de saúde física e psíquica.

Supõe-se que a diferenciação dos aspectos de saúde física divergiu das probabilidades de nível mental, em certa medida, por questões relacionadas ao perfil do gestor do sistema socioeducativo. Culturalmente, ainda fruto da consequência do processo não constituído (enraizado) da identidade do socioeducador (BISINOTO, 2017), atribui-se, a crença da necessidade de valores ligados ao vigor e à estética, para que, em casos excepcionais - realizem atividades que careçam de força física para contenção e segurança (BAPTISTA, 2013). 


\section{Revista \\ Debates Insubmissos}

Sob a perspectiva do desenvolvimento de burnout (MASLACH, 2003), a taxa mais elevada dos gestores (de esgotamento emocional - MBI-EE para $70 \%$ dos participantes desta pesquisa) interferiu na saúde mental deles devido a um estado emocional defasado (correlação= -0,5282). Dentro dessa ótica, o 'esgotamento emocional' (MBI-EE) demonstrouse inversamente proporcional ao nível de 'saúde mental' (SF-36). Assim, deduziu-se, sob os aspectos do $S F-36$, em especial quando somados à avaliação referente aos aspectos de saúde mental, que esta área merece atenção especial ao referir-se ao autocuidado.

Sob os domínios de saúde ( $S F$-36), o escore médio de 'capacidade funcional' (CF) revelou uma taxa significativamente maior que o índice do domínio de 'vitalidade' (VT), sendo o nível de escore médio deste o mais baixo dos oitos domínios em ambos os Grupos alvos de investigação.

Sob o ponto de vista da saúde mental, verificou-se que o nível de burnout relacionado ao 'esgotamento emocional' e ao aspecto da 'vitalidade' ( $S F-36, \mathrm{VT}$ ) ocorreu sob relação de proporções inversas, ou seja, quanto maior o domínio da 'vitalidade' (SF-36, VT), menor o nível de 'esgotamento emocional' (MBI-EE).

Os resultados também apontaram que parte considerável dos gestores $(36 \%)$ apresentaram as taxas mais baixas de 'vitalidade' ( $S F-36, V T)$ e estão sob tratamento clínico psiquiátrico. Em relação ao campo socioambiental, a grande maioria (93\%) dos que se mostraram com o domínio de 'vitalidade' baixo mencionaram os aspectos físicos no ambiente organizacional como prejudiciais no contexto de saúde referente ao trabalho.

Sob este ângulo, é notório ainda que os gestores que obtiveram índices baixos em relação à qualidade de vida $(S F-36)$ apresentaram maiores dificuldades na execução das atividades diárias, devido aos riscos encontrados no ambiente de trabalho. Em contrapartida, conforme os resultados expostos acima, aqueles que revelaram que nunca se estressaram ao lidar diretamente com pessoas (MBI-HSS, item 16) indicaram saúde mental mais fortalecida.

Nesse sentido, consoante à pesquisa de Souza (2012), verificou-se neste estudo que o contexto de trabalho interferiu significativamente nas vivências de saúde dos gestores da 


\section{Revista \\ Debates Insubmissos}

socioeducação, sinalizando a precariedade das condições e dos meios de trabalho a partir da queda da qualidade de vida no ambiente de trabalho, bem como sobre os principais 'sintomas' percebidos fora da unidade socioeducativa.

Para as pesquisas conseguintes, de acordo com a perspectiva da Organização Mundial de Saúde (WHOQOL GROUP, 1994) acerca da prospecção de uma qualidade de vida considerada 'saudável', este estudo indicou que há a necessidade de identificação e descrição dos fatores ligados à saúde física, ao estado psicológico, ao nível de independência e às relações sociais do indivíduo e suas interfaces com o meio onde está inserido, e se estes estão correlacionados à baixa saúde mental experimentada pelos gestores - de acordo com o resultado do nível MCS, SF-36 (pontuação de 24,54). Além disso, há a urgência de verificar, conforme Ware et al. (1994), quais fatores negativos (síndromes ou doenças) estão concomitantes a uma saúde mental não saudável.

Por outro lado, de forma também relevante, é importante averiguar os aspectos positivos relacionados ao bem-estar, pois esses podem legitimar a ressignificação de modos e estilos de vida assim como a contínua construção e atualização de propostas que visem a saúde do trabalhador que gerencia a socioeducação no Distrito Federal.

Sumariamente, este estudo sobre a avaliação da saúde física e emocional, a partir do $S F$ 36 e MBI-HSS forneceu subsídios para a criação de programas de saúde pública destinados aos profissionais que atuam ementidades governamentais, especialmente àqueles que realizam atendimento com altos níveis de complexidade em termos psicológicos, emocionais e de sobrecarga de trabalho.

Há de se considerar que, para se realizar um panorama de saúde sob o ponto de vista holístico e da teoria organísmica, como propõe Ribeiro (2019), faz-se vital analisar outros aspectos para além do contexto de trabalho. Como identificados neste artigo, $66 \%$ dos gestores dedicavam-se a atividades domésticas; $37 \%$ às de educação dos filhos; $31 \%$ realizavam outros cursos; $18 \%$ trabalhavam em mais de um emprego; e 60\% estavam 'parcialmente satisfeitos' com as vivências de lazer, cultura, e de atividades esportivas e/ou artísticas. Em especial, é relevante focar na autopercepção sobre as formas de se estar inserido e integrado (no sentido 


\section{Revista \\ Debates Insubmissos}

relacional), de fazer contatos para, neste contexto, ressaltar a necessidade do movimento direcionado à criação de estratégias de enfrentamento, de mediação individual e coletiva sob a perspectiva de harmonização da saúde em seu conceito 'biopsicossociotranscendental' (RIBEIRO, 2019; FERREIRA, MENDES, 2003).

De forma paralela, esta pesquisa oportunizou o início de um processo que possibilita a revisão e a atualização de escolhas que integrem as respectivas vivências fora da organização relacionadas à qualidade de vida - às necessidades individuais percebidas e apontadas por cada gestor, em especial àqueles que ainda não manifestaram interesse e/ou não acessaram outras formas de autocuidado relacionado à saúde mental.

Sob a ótica humanista e existencial, em que a autonomia e a liberdade de escolha viabilizam processos de harmonização individual, em consonância com os aspectos geobiológicos e sacrotranscendentais sob as formas de fazer contato - no sentido relacional e abrangente da saúde (RIBEIRO, 2019) -, faz-se de extrema relevância a investigação, em próximas pesquisas, das necessidades específicas relacionadas ao trabalho na gestão, as quais os questionários aqui referenciados (MBI-HSS e $S F-36)$ não contemplaram por limitações de abrangência às especificadas da realidade vivida no sistema socioeducativo, quer sejam sóciohistóricas, quer sejam territoriais e/ou culturais.

Nessa direção, sugere-se a realização de novos estudos relacionados à qualidade de vida e ao contexto de trabalho de forma complementar a utilização de análises qualitativas descritivas e inferenciais (análise de conteúdo, de discurso e hermenêutica), tendo em vista que a diversificação da técnica de coleta e análise de dados é de grande valia quando o objeto de estudo é a inter-relação 'indivíduo-trabalho-saúde'.

Dessa forma, os resultados que foram apresentados puderam fornecer valiosas indicações empíricas, sinalizando que se trata de um tema que merece cuidado em um projeto de mudanças institucionais. Atenção especial deve ser dedicada à promoção da qualidade de vida caso se deseje agir preventivamente para evitar mais efeitos negativos, e a médio e longo prazos quanto à saúde dos gestores e a efetividade do trabalho. 
Para maior representatividade, em estudos seguintes sugere-se a realização de divulgaçõesacerca da importância da participação em pesquisas em contextos além do universo científico com a intenção de desenvolver, no campo socioeducativo, o protagonismo substancial e representativoda população em geral, que de fato atenda às reais carências de quem gerencia e executa a tão invisibilizada política pública da socioeducação.

Sob a ótica do método de amostragem voluntária - não probabilística -, deve-se ponderar que este estudo, em seu Termo de Consentimento Livre e Esclarecido, apresentou a característica de voluntariedade para o preenchimento dos instrumentais. Por consequência, essa informação pôde indicar um 'risco de resposta' (OLIVEIRA et al., 2019), uma vez que os respondentes foram selecionados conforme a vontade e o conhecimento de cada participante para responder a pesquisa.

Com isso, apreendeu-se que a não identificação do estado de saúde dos gestores, dentre outros fatores não previsíveis como o conteúdo, a extensão dos questionários e o tempo dispendido para o preenchimento de todos os 105 itens da pesquisa, remeteu-se para a importância de um olhar de maior amplitude, a priori imensurável, sobre os gestores que não puderam e/ou quiseram acessar a pesquisa ou finalizar as respostas dos instrumentos utilizados na pesquisa. O fato de que 103 gestores iniciaram e não terminaram o preenchimento dos questionários fortaleceu a reflexão de que, em pesquisas futuras, a análise de dados seja quantiqualitativa.

Uma outra valiosa e fundamental questão a ser realçada para trabalhos futuros é a urgência de um estudo aprofundado que identifique, descreva e discuta as desigualdades sob uma perspectiva interseccional de gênero, abordando classe e raça (ALVIM, 2020) de modo que o perfil do grupo de gestores perpasse pelo exercício de uma construção de propostas que abarquem as semelhanças e as diferenças, de acordo com a realidade apresentada nesse contexto. Homens e mulheres diferem tanto na forma de desencadear o desenvolvimento de burnout quanto na maneira de lidar com os fatores associados à qualidade de vida. Além disso, existem também fatores discriminatórios, em contexto social e de trabalho, dominados por regras que privilegiam os interesses dos homens (ZANELLO, 2018). Sob esse contexto de 
constituição identitária sócio-histórico, há de se investigar no universo socioeducativo se há e, caso a resposta seja positiva, como acontece o empoderamento de gênero.

Aliadas a outras pesquisas, urge a necessidade de que propostas de valorização da saúde, especialmente para aqueles que lidam com um contexto de alto nível de pressão (gestão), possam surgir de forma a reverberar não só na saúde mental e na qualidade de vida dos servidores públicos, mas também, por consequência, como direitos humanos e cidadania, na qualidade, na eficiência e na eficácia do serviço público prestado.

Por fim, espera-se contribuir com outros estudos no campo da saúde, psicopatologia e contexto organizacional de forma a trazer luz para processos que busquem a primazia da ética e do respeito à dignidade humana para fortalecer relações socioprofissionais, contrapondo-se aos modelos de gestão em que imperam as relações cristalizadas de poder. O intuito majoritário é politizar a ação destinada ao gestor, com foco em um ambiente que ofereça qualidade de vida e experiências mais saudáveis na esfera da administração pública, em especial a da socioeducação no Distrito Federal.

\section{REFERÊNCIAS}

ALVIM, Mônica Botelho. O Estético-Político em Merleau-Ponty e a Fenomenologia Crítica: Notas Preliminares sobre Relações Raciais. Estudos e Pesquisas em Psicologia (Online), [s. L.], v. 20, n.4, 2020.

AMARAL, Letícia Toledo. Instituições de internação para adolescentes em conflito com a Lei: a inserção dos amigonianos em Brasília. 2008. Dissertação (Mestrado em Psicologia) Universidade de Brasília, Brasília, 2008.

BAPTISTA, Gustavo Camilo. Impacto das relações intersubjetividade na implementação da política nacionalde atendimento socioeducativo. 2013. Tese (Doutorado em Psicologia Clínica e Cultura) - Universidade de Brasília. Brasília, 2013.

BENEVIDES-PEREIRA, Ana Maria Teresa. Considerações sobre a síndrome de burnout e seu impacto no ensino. Bol. psicol., São Paulo, v. 62, n. 137, p. 155-168, dez. 2012. Disponível em: http://pepsic.bvsalud.org/scielo.php?script=sci_arttext\&pid=S000659432012000200005 \&lng= pt\& nrm=iso. Acesso em: 20 set. 2021. 
BION, Wilfred Ruprecht. Estudos psicanalíticos revisados (Second thoughts). Rio de Janeiro, Imago, 1998.

BISINOTO, Cynthia. Docência na socioeducação: a experiência de um processo de formação continuada. Brasília: FUP - UnB, 2017.

BISINOTO, Cynthia; OLIVA, Olga; ARRAES, Juliana; GALLI, Carolina; AMORIM, Gustavo; ALVES, Luana; et. al. Socioeducação:origem, significado e implicações para o atendimento socioeducativo. Psicologia em Estudo, [s. L.], v. 20, (4), 575-585, 2015.

BRASIL. Decreto-Lei no 2.848, de 7 de dezembro de 1940. Código Penal. Brasília: Planalto, 1940. Disponível em: http://www.planalto.gov.br/ccivil_03/decretolei/del2848compilado.htm. Acesso em: 20 set. 2021.

BRASIL. Constituição (1988). Constituição da República Federativa do Brasil. Brasília, DF: Senado Federal: Planalto, 1988. Disponível em http://www.planalto.gov.br/ccivil_03/constituicao/constituicao.htm. Acesso em: 20 set. 2021.

BRASIL. Lei no 8.069, de 13 de julho de 1990. Dispõe sobre o Estatuto da Criança e do Adolescente e dá outras providências. Brasília: Planalto, 1990. Disponível em: http://www.planalto.gov.br/ccivil_03/leis/18069.htm. Acesso em: 20 set. 2021.

BRASIL. Lei n. 12.594, de 18 de janeiro de 2012. Institui o Sistema Nacional de Atendimento Socioeducativo [...]. Brasília, DF: Planalto, 2012. Disponível em: http://www.planalto.gov.br/ccivil_03/_Ato2011-2014/2012/Lei/L12594.htm. Acesso em: 20 set. 2021.

BOWLING, Ann.; BRAZIER John. Quality of life in social science and medicine Introduction. Social Science \& Medicine, [s. L.], v. 41, 1337-1338, 1995.

CANGUILHEM, Georges. La Santé: concept vulgaire et question philosophique. Toulouse: Sables, 1990.

CODO, Wanderley; VASQUES-MENEZES, Ione. Síndrome de burnout em acs e estratégias de enfrentamento. Cadernos de Saúde do Trabalhador, [s. L.], 14, 29-48, 1999.

COUTINHO, Maria da Penha; FRANKEN, Ieda. Qualidade de vida no serviço público de saúde: representaçõessociais de profissionais da saúde. Psicologia: ciência e profissão, Brasília, v. 29, n. 3, p. 448-461, 2009. Disponível em http://pepsic.bvsalud.org/scielo.php?script=sci_arttext\&pid=S1414-989320090003 $00003 \&$ lng=pt\&nrm=iso. Acesso em: 20 set. 2021.

DISTRITO FEDERAL. Decreto n..$^{\circ}$ 37.896, de 27 de dezembro de 2016. Regimento Interno da Secretaria de Estado de Políticas para Crianças, Adolescentes e Juventude do Distrito Federal. Brasília: SINJ-DF, 2016. Disponível em: http://www.sinj.df.gov.br/sinj/Norma/8775d15583ad47b6a4dc 131a47bc9f30/Decreto37896 27122016.html. Acesso em: 20 set. 2021. 


\section{DISTRITO FEDERAL. Plano Decenal de Atendimento Socioeducativo do Distrito}

Federal. Portaria 163 de 14 de agosto de 2015. Decreto 35.697 de $1^{\circ}$ de agosto de 2015. Distrito Federal: Governo do Distrito Federal, 2016 Disponível em:

http://www.crianca.df.gov.br/wp-conteudo/uploads/2018/02/Plano-Decenal-de-AtendimentoSocioeducativo-do-DF.pdf. Acesso em: 20 set. 2021.

FERREIRA, Mário César; MENDES, Ana Magnólia. Trabalho e riscos de adoecimento: o caso dos auditores-fiscaisda Previdência Social brasileira. Brasília: Ler, Pensar e Agir, 2003.

FLECK, Marcelo Pio. O instrumento de avaliação de qualidade de vida da Organização Mundial da Saúde (WHOQOL-100): características e perspectivas. Ciênc. saúde coletiva, [s. L.], v. 5, n. 1., 2000. Disponível em: https://doi.org/10.1590/S1413-81232000000100004. Acesso em: 20 set. 2021.

JESUS, Vania Cristina Pauluk de. Condições escolares e laborais de adolescentes autores de atosinfracionais: um desafio à socioeducação. Revista eletrônica de educação, [s. L.], 7(3), p. 129-142, 2013.

LOBÃO, Maria João. O Conceito de Saúde e a Minha Prática da Medicina Interna. Lisboa. Revista SPMI, [s. L.], v. 26, no. 2, p. 153-155, 2019. Disponível em < https://revista.spmi.pt DOI:10.24950/rspmi/PV/MJL/2/2019>.

MARÇAL, Ely. Uma compreensão da subjetividade de adolescentes no contexto de uma instituição executora de medida socioeducativa de semiliberdade do DF. [Trabalho Final de Curso não publicado]. Universidade Católica de Brasília, Brasília, 2007.

MASLACH, Christina. Job Burnout: New directions in research and intervention. Current Directionsin Psychological Science, [s. L.], v. 12, n. 5, p. 189-192, 2003.

OLIVEIRA, Maria dos Remédios; SOARES, Rackynelly; SOUSA, Maria Fátima; MENDONÇA Ana Valéria; DELDUQUE, Maria Célia. Mediação como prevenção à judicialização da saúde: narrativas dos sujeitos do judiciário e da saúde. Escola Anna Nery, [s. L.], v. 23, n. 2, 2019. Disponível em: https://dx.doi.org/10.1590/2177-9465-ean2018-0363. Acesso em: 20 set. 2021.

R CORE TEAM. R: a language and environment for statistical computing. R Foundation for Statistical Computing, Vienna, Áustria, R-rproject, 2019. Disponível em: https://www.Rproject.org/. Acesso em: 20 set. 2021.

RIBEIRO, Jorge Ponciano. O ciclo do contato: temas básicos na abordagem gestáltica. 8. ed. São Paulo: Summus, 2019.

ROMANS, Marcelo Domingues; PETRUS, Antoni; TRILLA, Jaume. Profissão: educador social. Trad. Ernani Rosa. Porto Alegre: Artmed, 2003.

SCHAUFELI, Wilmar; MASLACH, Christina; MAREK, Tadeusz. (Eds). Professional Burnout: Recent developments in Theory and Research. Philadelphia, PA: Routledge, 2018. 
SCHAUFELI, Wilmar; GREENGLASS, Esther. Introduction to special issue on burnout and health. Psychology Health, [s. L.], v. 16, n. 5, p. 501-510, 2001.

SEIDL, Eliane Maria; ZANNON, Célia Maria. Qualidade de vida e saúde: aspectos conceituais e metodológicos. Cadernos de Saúde Pública, [s. L.], v. 20, n. 2, p. 580-588, 2004. Disponível em: https://doi.org/10.1590/S0102-311X2004000200027. Acesso em: 20 set. 2021.

SILVA, Clawdemy. A socioeducação e Direitos humanos: um estudo do projeto "A Arte do Saber". 2017. Dissertação (Mestrado em Direitos Humanos e Cidadania) - Universidade de Brasília, Brasília, 2017.

SOARES, Tatiana de Paula; SILVA, Roges; ANTUN, Abdallah. Leitura: A Arte do Saber: Proposta de (Re)integração Social a Adolescentes sob cumprimento de Medida Socioeducativa de Internação. [Projeto Piloto - Unidade de Internação de Santa Maria]. Certidão de Registro n. 673.264. da Fundação Biblioteca Nacional do Ministério da Cultura, RJ, 2014.

SOUZA, Jeane; URIO, Ângela; MANOROV, Maraisa; GEREMIA, Daniela; HILLESHEIM, Adriana; MADUREIRA, Valéria. Conceitos e práticas em saúde: a enfermagem comemorando o Dia Internacional da Saúde. Extensio: Revista Eletrônica de Extensão. 16. p. 123-132, 2019. Disponível em: https://doi.org/10.5007/1807-0221.2019v16n33p123. Acesso em: 20 set. 2021.

TAVARES, Marcelo. A clínica na confluência da história pessoal e profissional. In: CODO, Wanderley. (Org.). $O$ trabalho enlouquece? Um encontro entre a clínica e o trabalho. 1 ed. Petrópolis: Vozes, 2004. p. 53-103.

WARE, John. et al. The SF-36 health survey: development and use in mental health research and the IQOLA Project. International Journal of Mental Health, [s. L.], v. 23, n. 2, p. 4973, 1994. Disponível em: www.jstor.org/stable/41344687. Acesso em: 20 set. 2021.

WORLD HEALTH ORGANIZATION. Guidelines for the primary prevention of mental, neurological and psychosocial disorders: Staff Burnout. Geneva: Division of Mental Health World Health Organization, 1998.

WORLD HEALTH ORGANIZATION. World Health Organization. The Ottawa Charter for Health Promotion. Geneva: World Health Organization, 1986. Disponível em: https://www.who.int/healthpromotion/conferences/previous/ottawa/en/. Acesso em: 20 set. 2021.

WORLD HEALTH ORGANIZATION. WHO called to return to the Declaration of Alma-Ata. Geneva: World Health Organization, 2017. Disponível em: http:// www.who.int/social_determinants/tools/multimedia/alma_ata/en/. Acesso em: 20 set. 2021. 
WHOQOL GROUP. The development of the World Health Organization quality of life assessment instrument. In: ORLEY, John.; KUYKEN, Willem. (Eds.). Quality of life assessment: international perspectives. Heidelberg: Springer, 1994. p. 41-60.

SOUZA, Tatiana Yokoy de. Processos de desenvolvimento de educadores sociais do sistema de medidas socioeducativas: indicadores de formação. 210 p. Tese (Doutorado em Processos de Desenvolvimento Humano e Saúde) - Universidade de Brasília. Brasília, 2012.

ZANELLO, Valeska. Saúde Mental, Gênero e Dispositivos: Cultura e processos de subjetivação. Curitiba: Appris, 2018.

Recebido: 30/06/2021

Aprovado: 21/09/2021 